\title{
Article
}

\section{Conformal Array Geometry for Hemispherical Coverage}

\author{
Fortuna Munno (D)
}

check for

updates

Citation: Munno, F. Conformal Array Geometry for Hemispherical Coverage. Electronics 2021, 10, 903. https://doi.org/10.3390/

electronics10080903

Academic Editor: Francisco Falcone

Received: 18 February 2021

Accepted: 7 April 2021

Published: 10 April 2021

Publisher's Note: MDPI stays neutral with regard to jurisdictional claims in published maps and institutional affiliations.

Copyright: (C) 2021 by the author. Licensee MDPI, Basel, Switzerland. This article is an open access article distributed under the terms and conditions of the Creative Commons Attribution (CC BY) license (https:/ / creativecommons.org/licenses/by/ $4.0 /)$.
Department of Engineering, University of Campania “L. Vanvitelli”, 81031 Aversa, Italy; fortuna.munno@unicampania.it

\begin{abstract}
Conformal arrays may be a viable solution in many antenna applications requiring a wide angular coverage with sufficiently high directivity values, so it is worth comparing different $2 \mathrm{D}$ conformal array geometries to satisfy these requirements. To this end, first, the singular value decomposition (SVD) of the radiation operator is exploited to determine the maximum directivity values an array can reach in the whole observation domain. A numerical study based on the maximum directivity and, hence, on the SVD is then proposed to select the array geometry complying with some given requirements. Therefore, the performances achievable by some array geometries (a semi-circumference, a trapezoidal, and an angle array) are analyzed, and the one assuring a better hemispherical coverage is suggested. Furthermore, such an SVD-based study is usefully exploited to determine which panels of a multi-faceted array must be fed to reach some assigned specifications.
\end{abstract}

Keywords: conformal arrays; high directivity beams; radiation operator; singular value decomposition

\section{Introduction}

In the last decades, thanks to their electric beam scanning capability, phased array antennas have gained great attention and have been utilized in several military and civilian applications [1-7]. Many of these applications, such as satellite communications [8], tracking surveillance [9], telemetry tracking and commanding (TT \& C) systems [10], radio astronomy [11], and SATCOM applications [12], require a hemispherical scan coverage. Besides, the same need has raised over the last few years too, when the demand for high data rate services has led to the birth of the 5 th generation (5G) mobile communication. Indeed, the latter uses millimeter-wave bands with large spectrum resources that suffer from relatively severe propagation losses impairing communication performances [13]. Hence, high gain arrays able to mitigate this attenuation are needed and phased array antennas are usually proposed to realize both the base station and mobile device [14,15].

However, there exists an intrinsic limitation on the achievable angular coverage using a single planar phased array, since the beamwidth of a planar phased array antenna would increase as its beam directs away from broadside direction (scan limitations typically restricted to $\pm 60^{\circ}$ ), unavoidably leading to loss of gain and degradation of the side-lobelevel [16]. To overcome this limitation, many works have aimed at a hemispherical coverage by the combination of several planar phased array antennas: in [17], the optimal number of planar faces was determined by a comparison between different configurations in terms of the minimum number of elements, the smallest aperture diameter, the smallest variation in gain throughout the hemisphere, and the maximum reflected power; in [18-20] some choice criteria regarding multiple planar phased arrays arranged in pyramids or pyramidal frusta were presented.

Here, a different approach based on the Singular Value Decomposition (SVD) [21] of the radiation operator linking the radiating source to the far-field is proposed. The SVD, indeed, represents a useful mathematical tool applicable to many electromagnetic contexts. In particular, in [22-29], the singular spectrum of the radiation or scattering operator was studied to foresee the information content of the data; in [30], it was used to determine the role of the source parametrization; in [31], it was exploited to determine the number 
of independent data in the phase retrieval problems. From a numerical perspective, the Truncated SVD (SVD) is a widespread regularized scheme to perform the inversion [32]. However, except for the rectilinear source for which it is exactly determined [33] and other configurations where it can be approximately deduced [34], only a numeric evaluation of the SVD can be computed. Nevertheless, since it does not constitute a big computational effort for the analyzed configurations, a numerical routine is used to compute the SVD of the radiation operators.

A first study about the hemispherical coverage capabilities of some sources exploiting the singular functions was presented in [35], where two figures of merit computed on the basis of the SVD were introduced to establish the terms of the comparison. However, the discussion was mainly focused on continuous sources, and no analytical link of the used figures of merit with the maximum achievable directivity of the sources was pointed out. Besides, no optimization on the angle source was introduced.

Later, in [36], the geometric parameters of an angle source were optimized to guarantee a uniform coverage within a $\pi$ interval. Instead, in [37], the singular functions of the radiation operator were used to predict the maximum directivity a continuous source can reach on a full observation angle.

Here, the results shown in [37] are first extended to the case of antenna arrays and then are exploited in the elaboration of a numerical strategy for the choice of the optimal array geometry complying with some given requirements. In particular, with reference to a $2 \mathrm{D}$ configuration (i.e., invariant along one axis), the numerical analysis is developed to search for the conformal array returning the highest possible directivities within a $\pi$ interval. To this end, different support geometries are considered by also including the angle array with optimal geometric parameters. Furthermore, it is shown that it is possible to foresee the role played by each side of a multi-faceted array in the radiation of a high directivity beam and, accordingly, to determine which panels have to be turned on, so increasing the practical effectiveness of the approach.

The paper is divided as follows. In Section 2, the radiation operator is introduced. The maximum directivity achievable by an array is determined in Section 3; then, this result is applied to determine the array geometry satisfying some coverage requirements in Section 4. The conclusions end the paper.

\section{The Radiation Operator}

In this paper, the radiating properties of some conformal array configurations are analyzed for the 2D case. Accordingly, the array is made up of $N_{a}$ filamentary elements arranged along an arbitrary curve (the array support). Such array radiates in a homogeneous medium with wavenumber $\beta=2 \pi / \lambda$, where $\lambda$ denotes the wavelength.

The radiated field is angularly observed in the variable $\theta \in[-\pi, \pi]$, and, up to an inessential factor, it can be written as:

$$
E(\theta)=\sum_{m=1}^{N_{a}} I_{m} h\left(\theta-\theta_{N m}\right) e^{j \beta\left[x_{m} \sin \theta+z_{m} \cos \theta\right]}=\mathcal{D} \underline{I},
$$

where $\left(x_{m}, z_{m}\right)$ is the position of the $m$-th element, $I_{m}$ is its excitation coefficient (the $m$-th entry of the vector $\underline{I})$, and $h\left(\theta-\theta_{N m}\right)$ is its element factor.

The element factor is supposed as:

$$
h\left(\theta-\theta_{N m}\right)=\operatorname{rect}\left(\frac{\theta-\theta_{N m}}{\pi}\right) \cos \left(\theta-\theta_{N m}\right),
$$

with $\theta_{N m}$ being the direction orthogonal to the array support in $\left(x_{m}, z_{m}\right)$ (see Figure 1). 


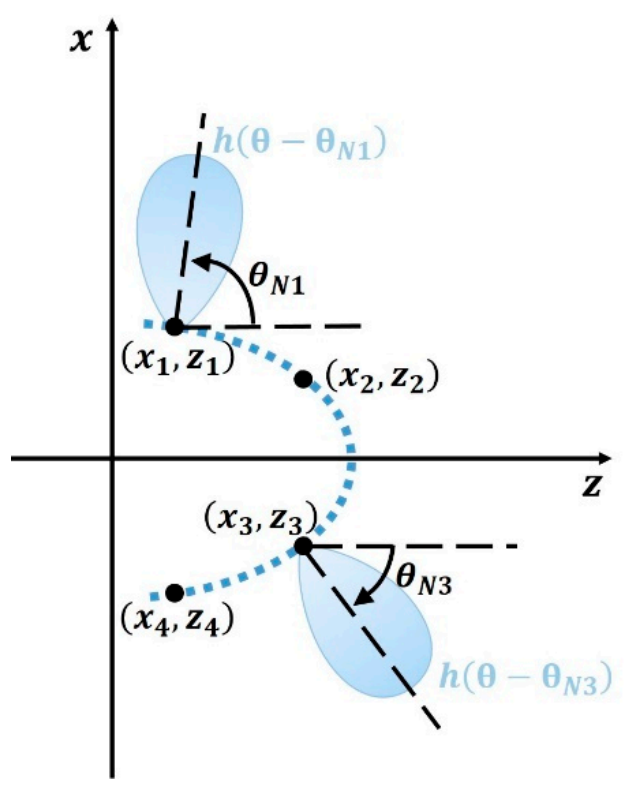

Figure 1. The geometrical setting of the array source: the $m$-th element location is $\left(x_{m}, z_{m}\right)$ with element factor $h\left(\theta-\theta_{\mathrm{Nm}}\right)$.

The operator $\mathcal{D}$ is a semi-discrete operator mapping the complex excitation vectors belonging to $\mathbb{C}^{N_{a}}$ to functions in $L_{[-\pi, \pi]}^{2}$, the latter being the subspace of finite energy functions supported over the interval $[-\pi, \pi]$.

As regards the analysis of the directive behavior of the arrays that will be developed in the following, the main mathematical tool exploited is the SVD of the operator, which consists of the right singular vectors $\underline{v}_{n}$ spanning the subspace in $\mathbb{C}^{N_{a}}$ of possible excitation coefficients vectors, the left singular functions $u_{n}(\theta)$ spanning the operator range, and the singular values $\sigma_{n}$. Another quantity involved in the analysis is the Number of Degrees of Freedom (NDF) [38,39]. For the particular case of compact operators, which $\mathcal{D}$ belongs to, there is a deep connection between the SVD of the operator and the NDF: the singular values of a compact operator accumulate to 0 and, accordingly, the NDF can be assumed as the number of the principal (i.e., above a fixed threshold) singular values of the relevant operator [40]. The importance of this quantity is twofold: on the one hand, it gives a measure of the number of "significant" and independent parameters needed to represent the radiated field, so that it can be identified as the dimension of the subspace of patterns a source (discrete, as for the excitation coefficients of an array, or continuous, when the source is represented by a current density function supported on a continuous domain) can radiate. On the other hand, it represents the minimum number of radiating elements of an array assuring the same patterns subspace dimension of the corresponding continuous source.

\section{The Maximum Directivity Function}

In [37], a directivity upper-bound for a continuous conformal source in the 2D space was found, and the expression of the field assuring the highest possible directivity was provided. Similarly to the continuous source case, when the source is discrete (as for the case of antenna arrays), the SVD of the corresponding semi-discrete operator can be introduced, and the orthonormality property of the left singular functions can be exploited for the determination of a directivity upper-bound.

For the following discussion, the directivity definition has been adapted to the bidimensionality of the configuration by considering the power radiated per unit plane angle. Once the directive gain is indicated by $D(\theta)$, for fields focusing in the $\theta_{0}$ direction, the directivity (which is the value of the directive gain at the direction of strongest emission) is given by $D\left(\theta_{0}\right)$ (see (A2) in Appendix A). Accordingly, what here is indicated as directivity 
upper-bound or maximum directivity $D_{\max }\left(\theta_{0}\right)$, should be intended as the directivity due to a particular field focusing in $\theta_{0}$ that maximizes it, that is:

$$
D_{\max }\left(\theta_{0}\right)=\max _{E \in L_{[-\pi, \pi]}^{2}} D\left(\theta_{0}\right) .
$$

The same manipulations reported in [37] on the directivity lead to the following directivity upper-bound:

$$
D\left(\theta_{0}\right) \leq 2 \pi \sum_{m=1}^{N D F}\left|u_{m}\left(\theta_{0}\right)\right|^{2}=D_{\max }\left(\theta_{0}\right),
$$

whose average value is related to the NDF according to the following relation

$$
\bar{D}_{\max }=\mathrm{NDF} .
$$

The latter coincides with the maximum directivity when $\left|u_{m}\left(\theta_{0}\right)\right|$ does not depend on $\theta_{0}$, for example, when the singular functions are complex exponentials.

Moreover, it can be shown that the field assuring the highest reachable directivity in the $\theta_{0}$ direction can be built as an expansion of left singular functions:

$$
\hat{E}(\theta)=\sum_{n=1}^{\mathrm{NDF}} u_{n}^{*}\left(\theta_{0}\right) u_{n}(\theta),
$$

where the expansion coefficients are set as

$$
c_{n}=u_{n}^{*}\left(\theta_{0}\right), n=1, \ldots, \mathrm{NDF} .
$$

Let us point out that the left singular functions can always be numerically computed for compact operators; hence, independently from the array geometry, the directivity upper bound and the corresponding radiated field can always be expressed as in (4) and (6).

Hereafter, confirmation of the effectiveness of (4) is provided by comparing it with the directivity due to a source excitation typically used in conformal array applications, i.e., the so-called phase compensation one. To this end, let us consider both a linear and semi-circumference array. For a linear array with semi-extension $a$ and center point $\left(x_{0}, z_{0}\right)$, the $m$-th element can be individualized by the arc length $s_{m}=(-a+(m-1) \Delta s)$ belonging to the interval $[-a, a]$, with $\Delta s=L /\left(N_{a}-1\right)$ and $L$ being the total length of the array (see Figure 2a). In cartesian coordinates, if $\phi_{0}$ is the angle formed by the array with the $z$-axis, the $m$-th element will be individualized by the couple $\left(x_{m}, z_{m}\right)=\left(x_{0}+s_{m} \sin \phi_{0}, z_{0}+s_{m} \cos \phi_{0}\right)$. As for the element factor (2), instead, $\theta_{N m}=\phi_{0}-\pi / 2 \forall m$. Finally, the combination of $M$ linear arrays can be studied by resorting to the superposition principle: the resulting field is simply the summation of the field contribution of each linear sub-array, whose parameters are identified by the subscript $i=1,2, \ldots, M$. 


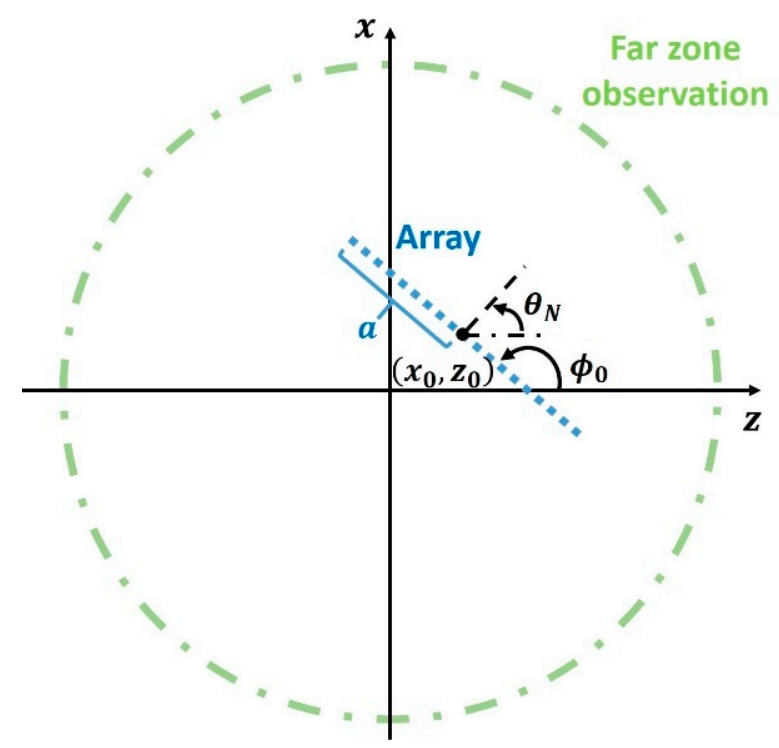

(a)

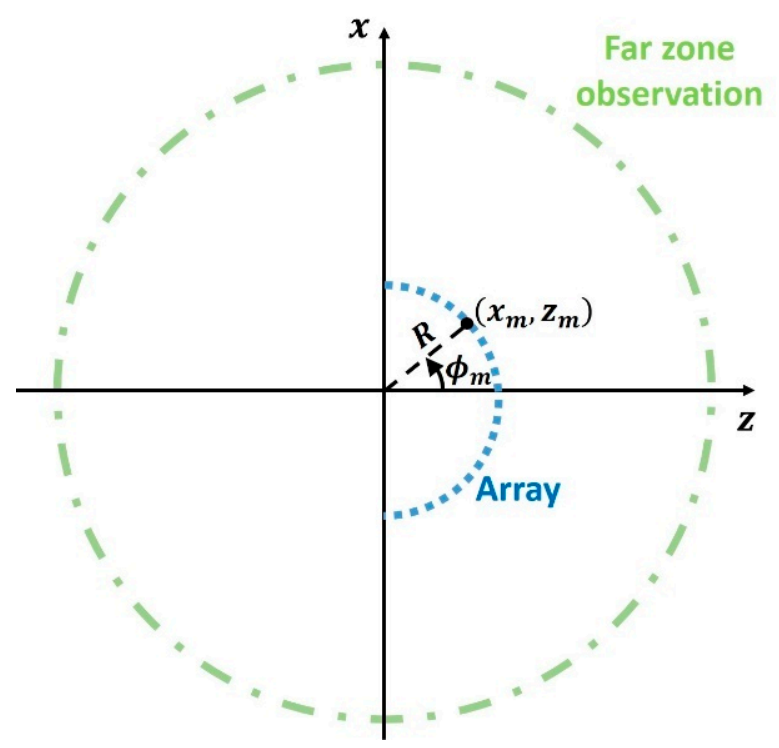

(b)

Figure 2. Parameters of a linear (a) and semi-circumference (b) array.

When a semi-circumference array of radius $R$ is considered (see Figure $2 b$ ), if the $N_{a}$ elements are uniformly distributed in angle, the $m$-th element is individualized by the angle $\phi_{m}=-\pi / 2+(m-1) \Delta \phi$ belonging to $[-\pi / 2, \pi / 2]$, with $\Delta \phi=\pi /\left(N_{a}-1\right)$. Accordingly, $\left(x_{m}, z_{m}\right)=\left(R \sin \phi_{m}, R \cos \phi_{m}\right)$, whereas the focusing direction of each element is $\theta_{N m}=\phi_{m} \forall m$.

A typical way to impose a beam focusing in a specific direction $\theta_{0}$ in conformal antenna design is by compensating the different path lengths characterizing the radiation from the source current elements to a reference plane orthogonal to the maximum direction [16], namely by choosing the excitation coefficients as:

$$
I_{m}=e^{-j \beta\left[x_{m} \sin \theta_{0}+z_{m} \cos \theta_{0}\right]} .
$$

When (8) is substituted in (1), the directivity expression is generally provided by:

$$
D\left(\theta_{0}\right)=\frac{N_{a}^{2}}{\sum_{m=1}^{N_{a}} \sum_{n=1}^{N_{a}} e^{-j \beta\left[\left(x_{m}-x_{n}\right) \sin \theta_{0}+\left(z_{m}-z_{n}\right) \cos \theta_{0}\right]} J_{0}\left(\beta d_{m n}\right)},
$$

(see Appendix A). The latter can be recast for the linear and semi-circumference cases, respectively, as:

$$
D\left(\theta_{0}\right)=\frac{N_{a}^{2}}{\sum_{m=1}^{N_{a}} \sum_{n=1}^{N_{a}} e^{-j \beta(m-n) \Delta \Delta s \sin \theta_{0}} J_{0}(\beta(m-n) \Delta s)},
$$

and

$$
D\left(\theta_{0}\right)=\frac{N_{a}^{2}}{\sum_{m=1}^{N_{a}} \sum_{n=1}^{N_{a}} e^{j 2 \beta R \sin \left(\theta_{0}-\frac{(n+m) \Delta \phi}{2}\right) \sin \left(\frac{(n-m) \Delta \phi}{2}\right)} J_{0}\left(2 \beta R \sin \left(\frac{(n-m) \Delta \phi}{2}\right)\right)} .
$$

Let us consider the simple example of an array with rectilinear or semi-circumference support of length $L=20 \lambda$, and $N_{a}=\mathrm{NDF}=41$ isotropic elements uniformly distributed. Then, the effectiveness of the upper-bound (4) is tested for both the geometries by comparing it with (10) and (11): in Figure 3, indeed, it is shown that the directivities values relative to the field produced by (8) (dashed red line) when changing the focusing angle are always less than or equal to the upper-bound (blue line), which represents the directivity 
value due to the field (6). In particular, when the linear array is considered (Figure 3a), the two curves almost overlap, meaning that the field due to (8) can be expanded as in (6), i.e., element excitations (9) provide the maximum directivity in a wide angular range.

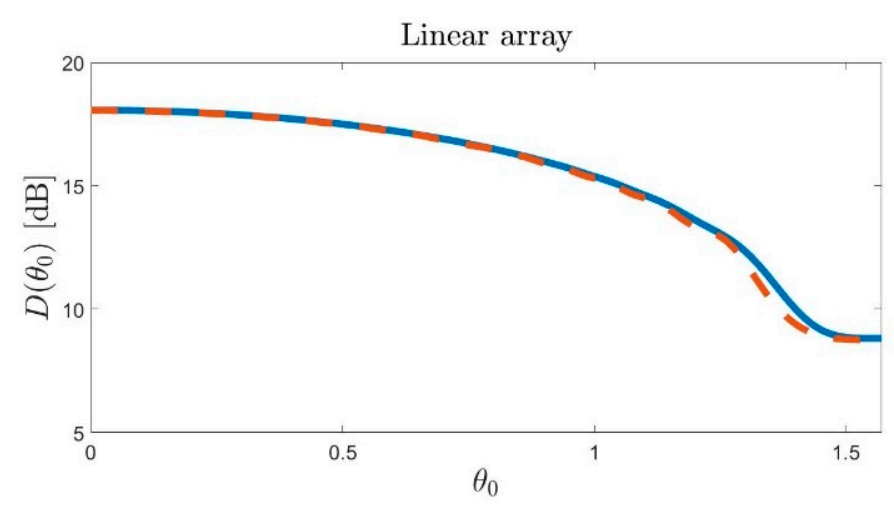

(a)

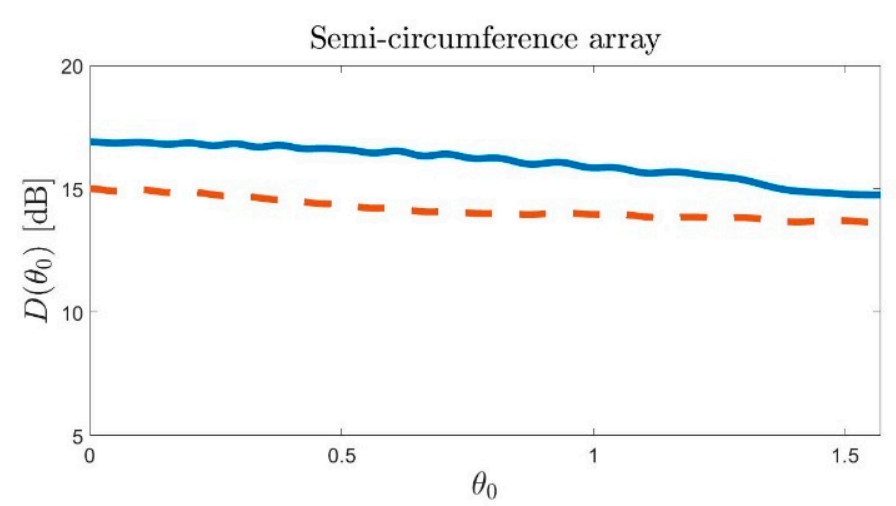

(b)

Figure 3. The directivity upper-bound (4) (blue line) and the directivities (10) and (11) (dashed red line), respectively, for a linear (a) and semi-circumference (b) array with 41 isotropic elements and length $L=20 \lambda$. For symmetry reasons, only the interval $[0, \pi / 2]$ is plotted.

\section{Application to Conformal Arrays}

This section shows that the results summarized above can be exploited to choose an array meeting certain assigned requirements.

Suppose it is needed to ensure that on an angular interval of $\pi$, the directivity values have a certain degree of uniformity and are greater than a given threshold, let say the mean directivity value (5). Intuitively, because of its geometric symmetry, one could think that a semi-circumference array is a good candidate for a $\pi$ coverage, expecting that arrays built with this shape or similar (for example, a trapezoidal shape made up of three segments) may represent a valid solution to the posed problem. Instead, on the basis of the results in Section 3, it will be shown that the angle array is the configuration that better meets the required specifications. Furthermore, it will be illustrated that it is not only possible to predict the performance of the array but also how to manage the activation of the panels to obtain good coverage when, for example, the object on which the antennas have to be mounted does not allow the use of the angle array considered here.

In order to compare the radiation properties of the arrays, the following parameters are introduced:

- $\quad$ the mean value of $D_{\max }$ computed on the $\mathrm{dB}$ curve only over the interval $[-\pi / 2, \pi / 2]$ and indicated by $\overline{\bar{D}}_{\max }$;

- the variance $\sigma^{2}$ of the $D_{\max }$ curve in $\mathrm{dB}$ with respect to $\overline{\bar{D}}_{\max }$ in the interval $[-\pi / 2, \pi / 2]$;

- $\quad$ the $D_{\max }$ range, $\Delta D_{\max }$, in the angular sector to cover;

- the maximum coverage angle $\theta_{\max }$, individualizing the angular interval $\left|\theta_{0}\right| \leq \theta_{\max }$ where $D_{\max }\left(\theta_{0}\right) \geq \bar{D}_{\max }$.

As for the number of elements $N_{a}$, it is chosen to assure the same patterns subspace dimension of the corresponding continuous source, namely, equal to the NDF. For a set of rectilinear sources and curved convex geometries, it is possible to estimate the NDF $[36,41]$ as approximately given by:

$$
\mathrm{NDF} \approx\left[\frac{2 L}{\lambda}\right]
$$

where $L$ is the source length.

Note that different array geometries with the same length have the same NDF. Moreover, looking at both (5) and (12), a direct link between $\bar{D}_{\max }$ and the support length can 
be deduced: different source geometries with the same length can reach the same mean directivity but different directive behavior point by point within the observation space.

Now, let us consider geometries with supports of the same length $L$. As a test case, $L$ is chosen equal to $20 \lambda$; accordingly, $N_{a}=\mathrm{NDF}=41$ and $\bar{D}_{\max }=16.13 \mathrm{~dB}$.

In the case of a semi-circumference array, since $L=20 \lambda$, the radius $R$ of the array must be set equal to $6.37 \lambda$ (blue stars of Figure 4).

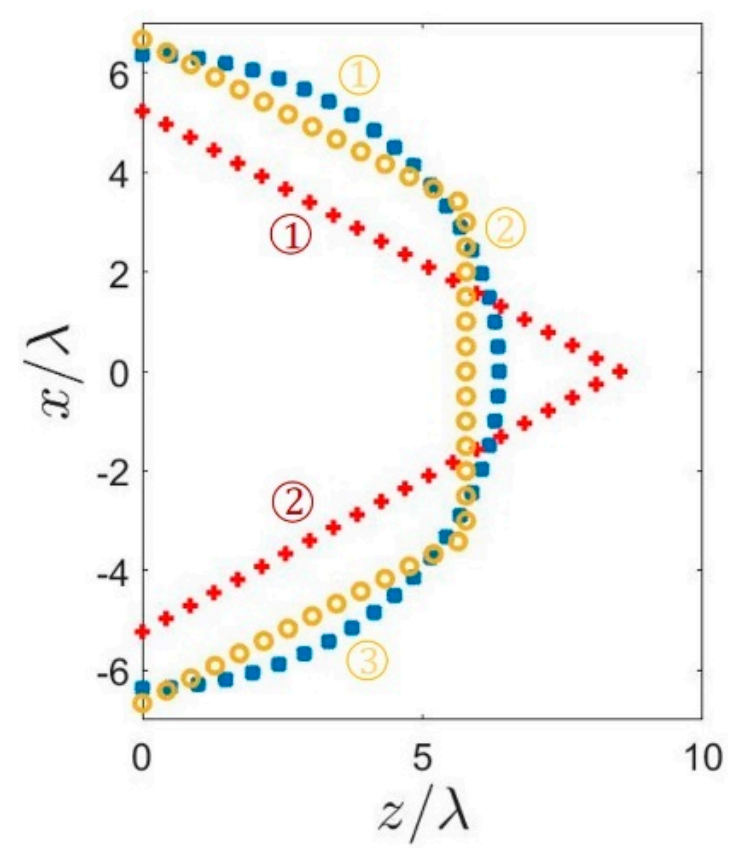

Figure 4. A semi-circumference array (blue stars), a trapezoidal array made of three linear arrays (yellow circles), and an angle array (red crosses) with the same number of elements. All the supports have the same length $L=20 \lambda$.

As can be observed in Figure $5, D_{\max }\left(\theta_{0}\right) \geq \bar{D}_{\max } \forall \theta_{0} \in[-\pi / 2, \pi / 2]$, as required. However, the corresponding directivity interval range is $2.79 \mathrm{~dB}$ with a variance $\sigma^{2}$ of 0.67 .

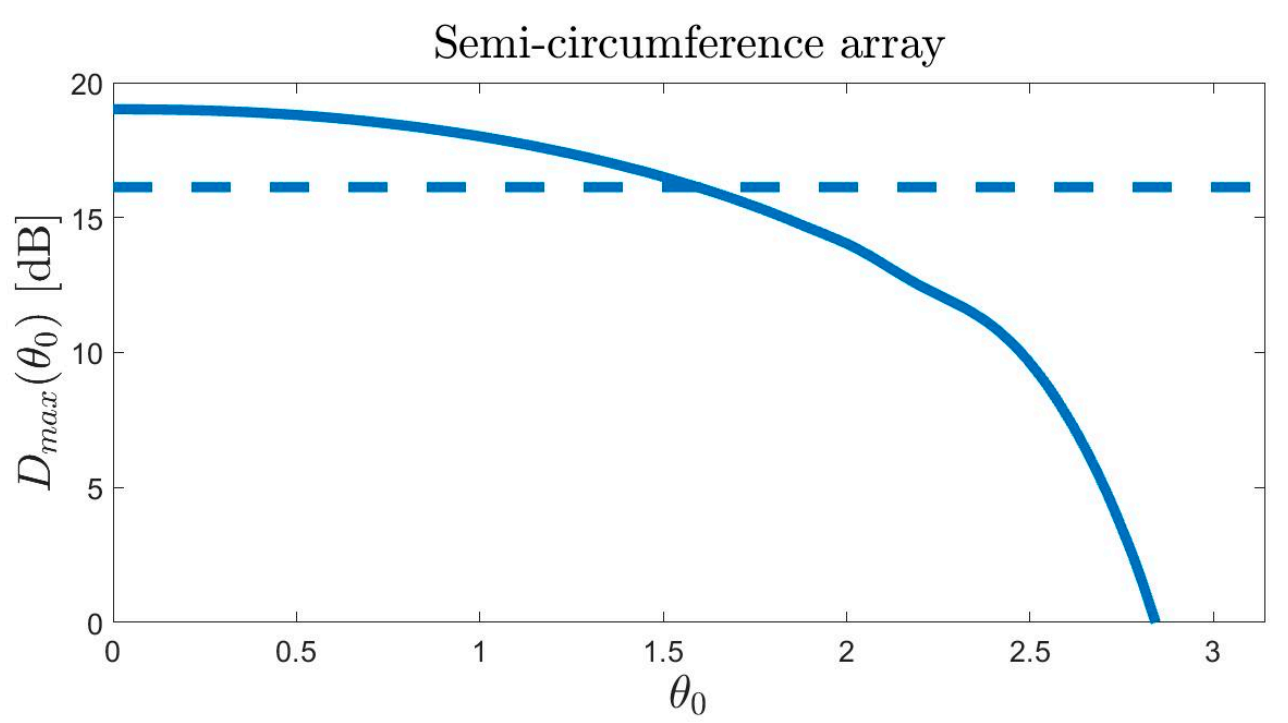

Figure 5. The behavior in $\mathrm{dB}$ of the maximum directivity $D_{\max }\left(\theta_{0}\right)$ of the semi-circumference array (solid blue line) in Figure 4. The dashed line represents the corresponding value of $\bar{D}_{\max }$. 
Then, let us see if one can do better by approximating the semi-circumference shape with three rectilinear sources (the trapezoidal shape of Figure 4).

To build an array of length $L$ with minimum discrepancy from a semi-circumference shape, the trapezoidal support must have segments of length $a_{1}=a_{2}=a_{3}=a=3.33 \lambda$ which form the angles $\phi_{01}=5 \pi / 6, \phi_{02}=\pi / 2, \phi_{03}=\pi / 6$ with the $z$-axis.

In Figure 6, the $D_{\max }$ curve relative to the case when all the three linear arrays are active (blue line) and to the case when only the arrays 1 and 2 are turned on (dotted red line) is reported (the one referring to the combined action of the arrays 2 and 3 is a mirror curve of the latter). As can be seen, the combined action of the three linear arrays has the effect of increasing the achievable directivity around the $\theta_{0}=0$ direction. However, even though the constrained hemispherical coverage is guaranteed, by keeping all the arrays active, $\Delta D_{\max }=3.30 \mathrm{~dB}$ and $\sigma^{2}=0.83$, namely, both the values are greater than the ones related to the semi-circumference array. Instead, if just one couple of arrays at a time is turned on (the couple $1-2$ for $0 \leq \theta_{0} \leq \pi / 2$ and $2-3$ for $-\pi / 2 \leq \theta_{0}<0$ ), $\Delta D_{\max }$ drops to $2.68 \mathrm{~dB}$ and $\sigma^{2}$ to 0.60 , resulting in a more uniform coverage in the half angular interval. Moreover, a further advantage of a selective activation is that not all the $N_{a}$ elements must be simultaneously fed.

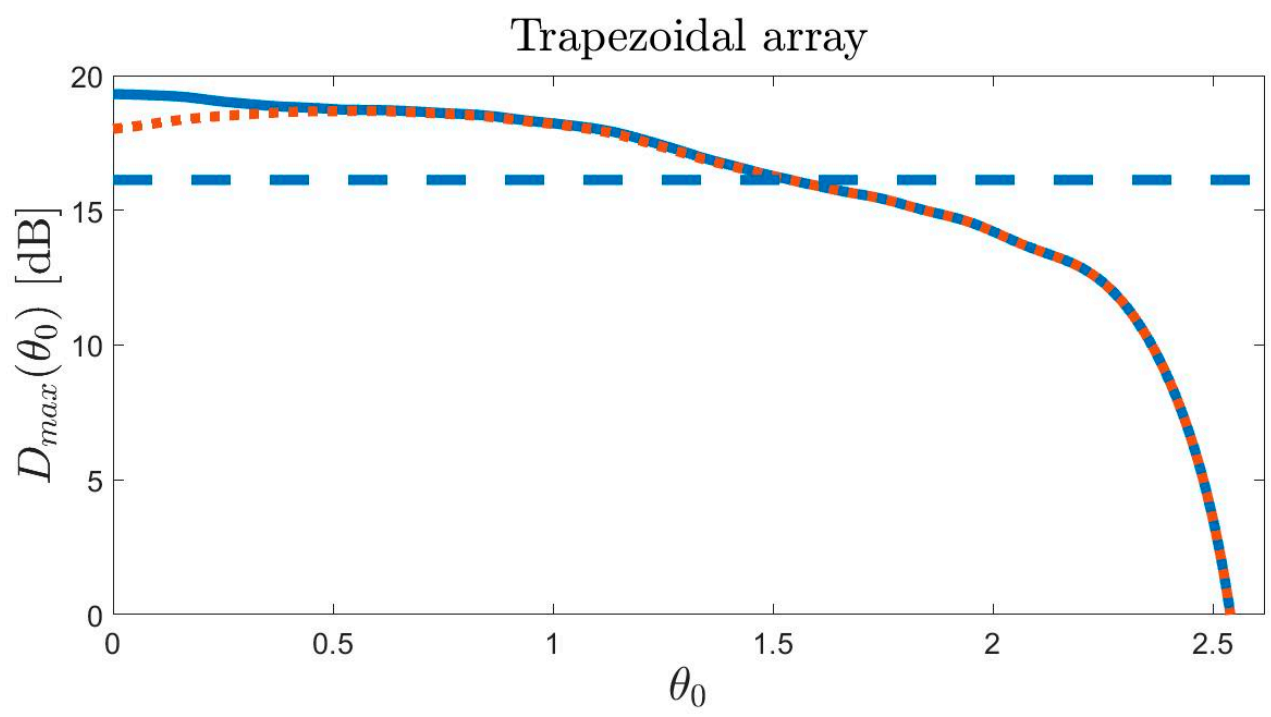

Figure 6. The behavior of the maximum directivity $D_{\max }\left(\theta_{0}\right)$ of the trapezoidal array (solid blue line) in Figure 4 and its corresponding value of $\bar{D}_{\max }$ (dashed blue line). The dotted red line represents the maximum directivity $D_{\max }\left(\theta_{0}\right)$ due to the only upper angle array forming the source, which specularly acts with respect to the lower angle array.

The price to pay is a small decrease of the average directivity value in this sector $\left(\overline{\bar{D}}_{\text {max }}=18.04 \mathrm{~dB}\right.$ for the trapezoidal array and $\overline{\bar{D}}_{\text {max }}=18.16 \mathrm{~dB}$ for the semi-circumference array) and non-rigorous compliance of the directivity constraint close to $\pm \pi / 2\left(\theta_{\max }=1.54 \mathrm{rad}\right)$.

Finally, let us consider an angle shape as in Figure 4, whose segments have a length $a_{1}=a_{2}=a=5 \lambda$ and form the angles $\phi_{01}=2.59 \mathrm{rad}, \phi_{02}=\pi-\phi_{01}$ with the $z$-axis. According to [36], such angles assure a uniform coverage for $\left|\theta_{0}\right| \leq \pi / 2$ with an element factor as in (2). From Figure 7, one can observe that, again, the effect of the combined action of both the linear arrays is to increase the directivity values around the 0 direction. However, differently from the trapezoidal case, it has the further effect to assure that $D_{\max } \geq \bar{D}_{\max }$ around 0 . Hence, the two linear arrays forming the source must be simultaneously fed; if only one array acts, the desired coverage requirements are not satisfied. As for the comparing parameters, $\Delta D_{\max }$ and $\sigma^{2}$ are significantly lower than those of the other array shapes. Accordingly, the angle array can radiate, on average, fields with the highest and 
most uniform values of directivity (fluctuating around $\overline{\bar{D}}_{\text {max }}=17.97 \mathrm{~dB}$ ) over a $\pi$ angular interval. Moreover, the observation interval where $D_{\max } \geq \bar{D}_{\max }$ is wider than the one of the previous cases, reaching a semi-extension of almost $2 \mathrm{rad}$. The values assumed by these parameters in all the analyzed cases are gathered in Table 1.

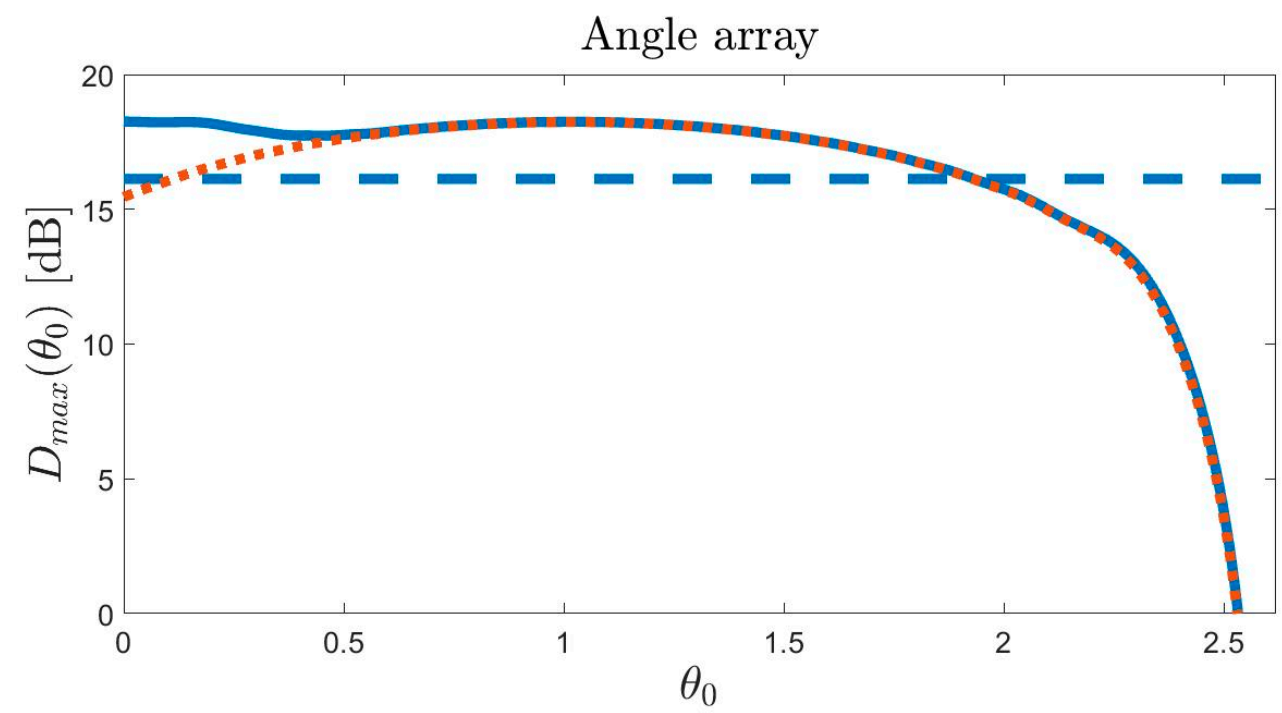

Figure 7. The behavior of the maximum directivity $D_{\max }\left(\theta_{0}\right)$ of the angle array (solid blue line) in Figure 4 and its the corresponding value of $\bar{D}_{\max }$ (dashed blue line). The dotted red line represents the maximum directivity $D_{\max }\left(\theta_{0}\right)$ due to the only upper linear array forming the source, which specularly acts with respect to the lower array. The dashed red line is the corresponding value of $\bar{D}_{\max }$.

Table 1. Table of the values of the parameters used to compare the arrays.

\begin{tabular}{ccccc}
\hline Array Shape & $\overline{\boldsymbol{D}}_{\max }[\mathrm{dB}]$ & $\sigma^{2}$ & $\boldsymbol{\Delta} \boldsymbol{D}_{\max }[\mathrm{dB}]$ & Coverage Angle [rad] \\
\hline Semi-circumference & 18.16 & 0.67 & 2.79 & 1.59 \\
Trapezoidal & 18.24 & 0.83 & 3.30 & 1.54 \\
Trapezoidal (sides 1-2 or & 18.04 & 0.60 & 2.68 & 1.54 \\
2-3 active) & 17.97 & 0.05 & 0.91 & 1.93 \\
Angle & &
\end{tabular}

In Figure 8, the high directivity fields built as in (6) for the three array configurations are reported with reference to the interval $[0, \pi / 2]$, since for the other half, there are symmetric results. The semi-circumference and trapezoidal arrays (with all the sides working together) follow similar trends with similar radiated fields, as expected since the two arrays are geometrically similar. When only two sides of the trapezoidal array are turned on, the field amplitude around 0 conforms to its value at $\pi / 4$, but at $\pi / 2$ the amplitude lowers, and the beam width enlarges. Instead, the angle array radiates a field with almost the same amplitude and beamwidth, even in the $\pi / 2$ direction. 

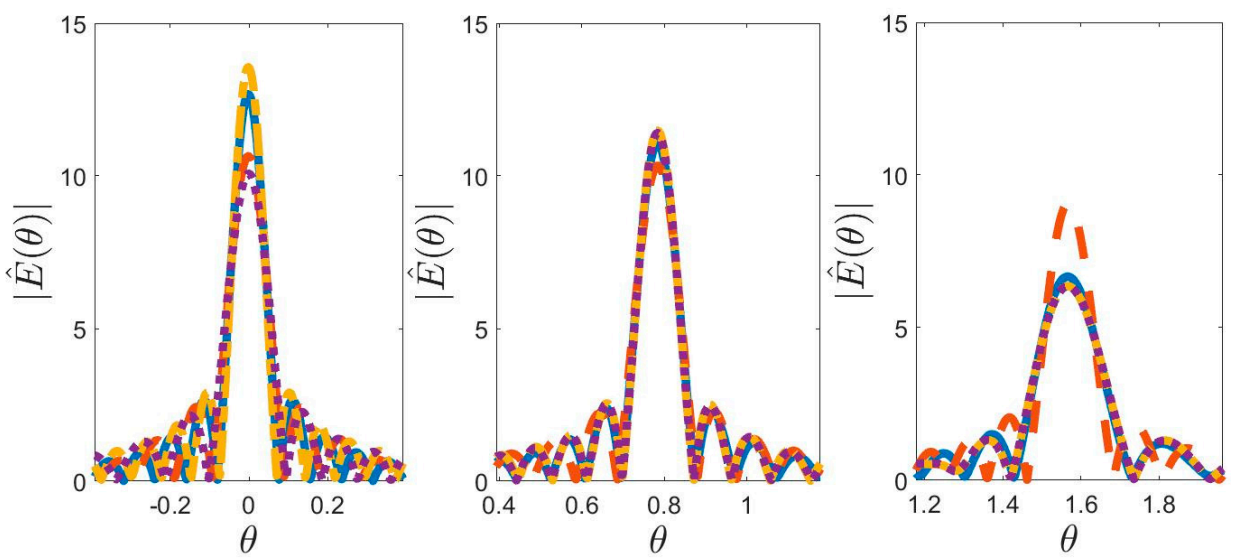

Figure 8. The amplitude of the field $\hat{E}(\theta)$ radiated by the semi-circumference array (solid blue lines), the trapezoidal array (dash-dotted yellow lines), the sides 1-2 of the trapezoidal array (dotted violet line), and the angle array (dashed red lines) of Figure 4 . The plots present the field amplitudes for the choice $L=20 \lambda$. From left to right, the three panels refer to the focusing angles $\theta_{0}=0, \pi / 4, \pi / 2$, respectively.

\section{Conclusions}

The SVD of the radiation operator, numerically computed, provides the basic elements (left singular functions and NDF) necessary for a deep analysis of the directive behavior of a conformal array. This paper has shown that the resulting knowledge about the maximum directivity can be exploited to select the array shape that better meets some prescribed requirements. In particular, the capability of a given array to guarantee a hemispherical coverage with directivity values greater than a certain threshold and with a certain degree of uniformity has been investigated. Concerning a 2D configuration, a semi-circumference, a trapezoidal, and an angle array with the same support length are compared using some parameters adequately introduced. The semi-circumference and trapezoidal arrays have similar behaviors; moreover, for the trapezoidal array, the maximum directivity study suggests that to feed a couple of sides at a time is sufficient to satisfy the requirements. However, contrary to what one would expect, the angle array with an optimized angle can provide better uniformity results: it guarantees the highest and most uniform values of directivity within the interval $[-\pi / 2, \pi / 2]$, and the wider coverage with directivities greater than the prescribed threshold.

Funding: This work was supported by the European Union and the Italian Ministry of University and Research funding under the "Programma Operativo Nazionale Ricerca e Innovazione 2014-2020".

Data Availability Statement: Not applicable.

Acknowledgments: The author wishes to thank Giovanni Leone and Rocco Pierri for their academic guidance and for providing many useful discussions.

Conflicts of Interest: The author declares no conflict of interest.

\section{Appendix A}

In this appendix, the mathematical steps to derive (9) are sketched. To do this, let us consider an array with elements distributed along a certain curve. In the case of isotropic elements (unitary element factor), when the excitation coefficients are chosen as in (8) to focus at the $\theta_{0}$ direction, the radiated field is:

$$
E(\theta)=\sum_{m=1}^{N_{a}} e^{j \beta\left[x_{m}\left(\sin \theta-\sin \theta_{0}\right)+z_{m}\left(\cos \theta-\cos \theta_{0}\right)\right]} .
$$

As for the directivity, since the geometry is invariant along one direction, the basic array element is a filamentary current, and the antenna theory results available in literature 
cannot be directly applied. However, by considering the power radiated per unit plane angle instead of the unit solid angle, the directivity definition can be adapted to the 2D case as:

$$
D\left(\theta_{0}\right)=\frac{\left|E\left(\theta_{0}\right)\right|^{2}}{\frac{1}{2 \pi} \int_{-\pi}^{\pi}|E(\theta)|^{2} d \theta}
$$

Then, by simply substituting (A1) into (A2), one obtains:

$$
D\left(\theta_{0}\right)=\frac{2 \pi N_{a}^{2}}{\int_{-\pi}^{\pi} \sum_{m=1}^{N_{a}} \sum_{n=1}^{N_{a}} e^{j \beta\left[\left(x_{m}-x_{n}\right)\left(\sin \theta-\sin \theta_{0}\right)+\left(z_{m}-z_{n}\right)\left(\cos \theta-\cos \theta_{0}\right)\right]} \mathrm{d} \theta} .
$$

By exchanging the integral and summation operations and moving out the constant term under the integral sign, the latter becomes:

$$
D\left(\theta_{0}\right)=\frac{2 \pi N_{a}^{2}}{\sum_{m=1}^{N_{a}} \sum_{n=1}^{N_{a}} e^{-j \beta\left[\left(x_{m}-x_{n}\right) \sin \theta_{0}+\left(z_{m}-z_{n}\right) \cos \theta_{0}\right]} \int_{-\pi}^{\pi} e^{j \beta\left[\left(x_{m}-x_{n}\right) \sin \theta+\left(z_{m}-z_{n}\right) \cos \theta\right]} \mathrm{d} \theta} .
$$

Observing that the phase function of the exponential inside the integral can be interpreted as the scalar product between the vector connecting the $m$-th and the $n$-th element and the observing direction, the integral can be recast as:

$$
\int_{-\pi}^{\pi} e^{j \beta\left[\left(x_{m}-x_{n}\right) \sin \theta+\left(z_{m}-z_{n}\right) \cos \theta\right]} \mathrm{d} \theta=\int_{-\pi}^{\pi} e^{j \beta d_{m n} \cos \alpha} \mathrm{d} \theta
$$

where $d_{m n}=\sqrt{\left(x_{m}-x_{n}\right)^{2}+\left(z_{m}-z_{n}\right)^{2}}$ and $\alpha$ is the angle between the difference vector and the observation angle direction. Since the integral (A5) is equal to $2 \pi J_{0}\left(\beta d_{m n}\right)$ [42], the directivity finally writes as:

$$
D\left(\theta_{0}\right)=\frac{N_{a}^{2}}{\sum_{m=1}^{N_{a}} \sum_{n=1}^{N_{a}} e^{-j \beta\left[\left(x_{m}-x_{n}\right) \sin \theta_{0}+\left(z_{m}-z_{n}\right) \cos \theta_{0}\right]} J_{0}\left(\beta d_{m n}\right)} .
$$

For a linear array with elements located along the $x$-axis $\left(\phi_{0}=\pi / 2\right)$, since $x_{m}=-a+(m-1) \Delta s$ and $z_{m}=0 \forall m$, Equation (A6) becomes:

$$
D\left(\theta_{0}\right)=\frac{N_{a}^{2}}{\sum_{m=1}^{N_{a}} \sum_{n=1}^{N_{a}} e^{-j \beta(m-n) \Delta s \sin \theta_{0}} J_{0}(\beta(m-n) \Delta s)} .
$$

However, a simpler expression can be derived when $2 a \gg \lambda$ and $\theta \cong \theta_{0}$. For a linear array, Equation (A4) reduces to:

$$
D\left(\theta_{0}\right)=\frac{2 \pi N_{a}^{2}}{\sum_{m=1}^{N_{a}} \sum_{n=1}^{N_{a}} e^{-j \beta(m-n) \Delta s \sin \theta_{0}} \int_{-\pi}^{\pi} e^{j \beta(m-n) \Delta s \sin \theta} \mathrm{d} \theta} .
$$

Let us focus, now, on the integral at the denominator. By exploiting the periodicity of the sine function, the integral is written as:

$$
\int_{-\pi}^{\pi} e^{j \beta(m-n) \Delta s \sin \theta} \mathrm{d} \theta=2 \int_{-\pi / 2}^{\pi / 2} e^{j \beta(m-n) \Delta s \sin \theta} \mathrm{d} \theta
$$

whereas the introduction of the variable $t=\sin \theta$ allows recasting it as

$$
2 \int_{-1}^{1} \frac{e^{j \beta(m-n) \Delta s t}}{\cos \theta} \mathrm{dt}
$$


Since the field in (A1) focuses in the $\theta_{0}$ direction, the main contribution to the integral comes from direction angles close to $\theta_{0}$. This allows a first approximation to be introduced into the denominator:

$$
\frac{2}{\cos \theta_{0}} \int_{-1}^{1} e^{j \beta(m-n) \Delta s t} \mathrm{dt}=\frac{4 \operatorname{sinc}(\beta(m-n) \Delta s)}{\cos \theta_{0}} .
$$

Accordingly, for a linear array:

$$
D\left(\theta_{0}\right) \simeq \frac{\pi}{2} \frac{N_{a}^{2} \cos \theta_{0}}{\sum_{m=1}^{N_{a}} \sum_{n=1}^{N_{a}} e^{-j \beta(m-n) \Delta s \sin \theta_{0}} \operatorname{sinc}(\beta(m-n) \Delta s)} .
$$

When $\Delta s=\lambda / 2$, (A12) becomes

$$
D\left(\theta_{0}\right) \simeq \frac{N_{a} \pi \Delta \cos \theta_{0}}{2}
$$

which resembles the corresponding 3D case of isotropic point sources.

Instead, if the array has a semi-circumference geometry, the expression of the directivity cannot be simplified and, taking into account that $d_{m n}=2 R \sin \left(\frac{\phi_{n}-\phi_{m}}{2}\right)$, it is written as:

$$
D\left(\theta_{0}\right)=\frac{N_{a}^{2}}{\sum_{m=1}^{N_{a}} \sum_{n=1}^{N_{a}} e^{j 2 \beta R \sin \left(\theta_{0}-\frac{(n+m) \Delta \phi}{2}\right) \sin \left(\frac{(n-m) \Delta \phi}{2}\right)} J_{0}\left(2 \beta R \sin \left(\frac{(n-m) \Delta \phi}{2}\right)\right)} .
$$

\section{References}

1. Weber, M.E.; Cho, J.Y.N.; Herd, J.S.; Flavin, J.M.; Benner, W.E.; Torok, G.S. The next-generation multimission U.S. surveillance radar network. Bull. Am. Meteorol. Soc. 2007, 88, 1739-1751. [CrossRef]

2. Zrnic, D.S.; Kimpel, J.F.; Forsyth, D.E.; Shapiro, A.; Crain, G.; Ferek, R.; Heimmer, J.; Benner, W.; Mcnellis, F.T.J.; Vogt, R.J. Agile-beam phased array radar for weather observations. Bull. Am. Meteorol. Soc. 2007, 88, 1753-1766. [CrossRef]

3. Zhang, G.; Doviak, R.J.; Zrnic, D.S.; Crain, J.; Staiman, D.; Al-Rashid, Y. Phased array radar polarimetry for weather sensing: A theoretical formulation for bias corrections. IEEE Trans. Geosci. Remote Sens. 2009, 47, 3679-3689. [CrossRef]

4. Fulton, C.; Yeary, M.; Thompson, D.; Lake, J.; Mitchell, A. Digital phased arrays: Challenges and opportunities. Proc. IEEE 2016, 104, 487-503. [CrossRef]

5. Herd, J.S.; Conway, M.D. The evolution to modern phased array architectures. Proc. IEEE 2016, 104, 519-529. [CrossRef]

6. Cheng, X.; Aubry, A.; Ciuonzo, D.; De Maio, A.; Wang, X. Robust waveform and filter bank design of polarimetric radar. IEEE Trans. Aerosp. Electron. Syst. 2017, 53, 370-384. [CrossRef]

7. Hamberger, G.F.; Späth, S.; Siart, U.; Eibert, T.F. A Mixed Circular/Linear Dual-Polarized Phased Array Concept for Automotive Radar-Planar Antenna Designs and System Evaluation at 78 GHz. IEEE Trans. Antennas Propag. 2019, 67, 1562-1572. [CrossRef]

8. Haupt, R. Optimizing an Antenna Array for Satellite Communications. In Proceedings of the Symposium Series on Computational Intelligence, Cape Town, South Africa, 7-10 December 2015; pp. 1170-1173.

9. ABM Research and Development at Bell Laboratories: Project History. Bell Laboratories (1975). Available online: http:/ www. srmsc.org/ref1020.html (accessed on 1 September 2000).

10. Anandan, V.K.; Pradeep, K.C.; Satyanarayana, S.N.V.; Sarkar, M. Multiple satellite telemetry and tracking system (MuST). In Proceedings of the IEEE Indian Conference on Antennas and Propagation, Hyderabad, India, 16-19 December 2018; pp. 1-4.

11. Jamnejad, V.; Huang, J.; Levitt, B.; Pham, T.; Cesarone, R. Array antennas for JPL/NASA deep space network. In Proceedings of the IEEE Aerospace Conference, Big Sky, MT, USA, 9-16 March 2002; Volume 2, pp. 911-921.

12. Malyuskin, O.; Fusco, V. Pointing accuracy and gain reduction mechanisms in CP retrodirective arrays for SATCOM applications. In Proceedings of the European Conference on Antennas and Propagation, Prague, Czech Republic, 26-30 March 2012; pp. 825-826.

13. Sulyman, A.I.; Nassar, A.T.; Samimi, M.K.; Maccartney, G.R.; Rappaport, T.S.; Al-sanie, A. Radio propagation path loss models for $5 \mathrm{G}$ cellular networks in the $28 \mathrm{GHz}$ and $38 \mathrm{GHz}$ millimeter-wave bands. IEEE Commun. Mag. 2014, 52, 78-86. [CrossRef]

14. Rupakula, B.; Zihir, S.; Rebeiz, G.M. Low complexity 54 63-GHz transmit/receive 64-and 128-element 2-D-scanning phasedarrays on multilayer organic substrates with 64-QAM 30-Gbps data rates. IEEE Trans. Microw. Theory Technol. 2019, 67, 5268-5281. [CrossRef]

15. Bang, J.; Choi, J. A SAR reduced mm-wave beam-steerable array antenna with dual-mode operation for fully metal-covered 5G cellular handsets. IEEE Antennas Wirel. Propag. Lett. 2018, 17, 1118-1122. [CrossRef] 
16. Josefsson, L.; Persson, P. Conformal Array Antenna Theory and Design; John Wiley \& Sons: Hoboken, NJ, USA, 2006 ; Volume 29.

17. Knittel, G. Choosing the number of faces of a phased-array antenna for hemisphere scan coverage. IEEE Trans. Antennas Propag. 1965, 13, 878-882. [CrossRef]

18. Kmetzo, J. An analytical approach to the coverage of a hemisphere by N planar phased arrays. IEEE Trans. Antennas Propag. 1967, 15, 367-371. [CrossRef]

19. Khalifa, I.; Vaughan, R. Geometric design of pyramidal antenna arrays for hemispherical scan coverage. IEEE Trans. Antennas Propag. 2007, 55, 468-471. [CrossRef]

20. Khalifa, I.; Vaughan, R. Geometric design and comparison of multifaceted antenna arrays for hemispherical coverage. IEEE Trans. Antennas Propag. 2009, 57, 2608-2614. [CrossRef]

21. Bertero, M. Linear inverse and ill-posed problems. Adv. Electron. Electron. Phys. 1989, 75, 1-120.

22. Solimene, R.; Maisto, M.A.; Pierri, R. Role of diversity on the singular values of linear scattering operators: The case of strip objects. J. Opt. Soc. Am. A 2013, 30, 2266-2272. [CrossRef] [PubMed]

23. Goodman, J.W. Introduction to Fourier Optics; Roberts and Company Publishers: Englewood, CO, USA, 2005.

24. Solimene, R.; Maisto, M.A.; Romeo, G.; Pierri, R. On the singular spectrum of the radiation operator for multiple and extended observation domains. Int. J. Antennas Propag. 2013, 2013. [CrossRef]

25. Solimene, R.; Mola, C.; Gennarelli, G.; Soldovieri, F. On the singular spectrum of radiation operators in the non-reactive zone: The case of strip sources. J. Opt. 2015, 17, 025605. [CrossRef]

26. Dell'Aversano, A.; Leone, G.; Ciaramaglia, F.; Solimene, R. A Strategy for Reconstructing Simple Shapes From Undersampled Backscattered Data. IEEE Geosci. Remote Sens. Lett. 2016, 13, 1757-1761. [CrossRef]

27. Brancaccio, A.; Dell'Aversano, A.; Leone, G.; Solimene, R. Subsurface detection of shallow targets by undersampled multifrequency data and a non-cooperative source. Appl. Sci. 2019, 9, 5383. [CrossRef]

28. Maisto, M.A.; Solimene, R.; Pierri, R. Valid angle criterion and radiation pattern estimation via singular value decomposition for planar scanning. IET Microw. Antennas Propag. 2019, 13, 2342-2348. [CrossRef]

29. Akbari Sekehravani, E.; Leone, G.; Pierri, R. NDF of Scattered Fields for Strip Geometries. Electronics 2021, 10, 202. [CrossRef]

30. Leone, G.; Munno, F.; Pierri, R. Radiation properties of conformal antennas: The elliptical source. Electronics 2019, 8, 531. [CrossRef]

31. Moretta, R.; Pierri, R. Performance of Phase Retrieval via Phaselift and Quadratic Inversion in Circular Scanning Case. IEEE Trans. Antennas Propag. 2019, 67, 7528-7537. [CrossRef]

32. Brancaccio, A.; Solimene, R. Fault detection in dielectric grid scatterers. Opt. Express 2015, 23, 8200-8215. [CrossRef]

33. Slepian, D.; Pollak, H.O. Prolate spheroidal wave functions, Fourier analysis and uncertainty-I. Bell Syst. Tech. J. 1961, 40, 43-63. [CrossRef]

34. Solimene, R.; Maisto, M.A.; Pierri, R. Sampling approach for singular system computation of a radiation operator. J. Opt. Soc. Am. A 2019, 36, 353-361. [CrossRef]

35. Leone, G. Source geometry optimization for hemispherical radiation pattern coverage. IEEE Trans. Antennas Propag. 2016, 64, 2033-2038. [CrossRef]

36. Leone, G.; Munno, F.; Pierri, R. Synthesis of Angle Arrays by the NDF of the Radiation Integral. IEEE Trans. Antennas Propag. 2021, 69, 2092-2102. [CrossRef]

37. Leone, G.; Munno, F.; Solimene, R. Field Synthesis of High Directivity Beams for Conformal Sources. IEEE Open J. Antennas Propag. 2021, 2, 439-452. [CrossRef]

38. Toraldo di Francia, G. Degrees of freedom of an image. J. Opt. Soc. Am. A 1969, 59, 799-804. [CrossRef]

39. Piestun, R.; Miller, D.A.B. Electromagnetic degrees of freedom of an optical system. J. Opt. Soc. Am. A 2000, 17, 892-902. [CrossRef]

40. Newsam, G.; Barakat, R. Essential dimension as a welldefined number of degrees of freedom of finite-convolution operators appearing in optics. J. Opt. Soc. Am. A 1985, 2, 2040-2045. [CrossRef]

41. Leone, G.; Munno, F.; Pierri, R. Inverse source on conformal conic geometries. IEEE Trans. Antennas Propag. 2021, 69, 1596-1609. [CrossRef]

42. Abramowitz, M.; Stegun, I.A. Handbook of Mathematical Functions with Formulas, Graphs, and Mathematical Tables; National Bureau of Standards: Washington, DC, USA, 1972. 